\title{
High-Quality Genome Resource of Clonostachys rosea Strain CanS41 by Oxford Nanopore Long- Read Sequencing
}

\author{
Haoming Wang, ${ }^{1}$ Yongrong Dong, ${ }^{1}$ Weixue Liao, ${ }^{1}$ Xin Zhang, ${ }^{1}$ Qinhu Wang, ${ }^{1}$ Guoqing Li, ${ }^{2}$ \\ Jin-Rong $\mathrm{Xu}^{3}$ and Huiquan Liu ${ }^{1, \dagger}$ \\ ${ }^{1}$ State Key Laboratory of Crop Stress Biology of Arid Areas and NWAFU-Purdue Joint Research \\ Center, College of Plant Protection, Northwest A\&F University, Yangling, Shaanxi 712100, China \\ ${ }^{2}$ College of Plant Science and Technology, Huazhong Agricultural University, Wuhan, 430070, China \\ ${ }^{3}$ Department of Botany and Plant Pathology, Purdue University, West Lafayette, IN 47907, U.S.A.
}

\section{Funding}

This work was supported by grants from the National Key R\&D Program of China (2019YFD1000605) and the National Youth Talent Support Program (Z111021802) to HL.

\section{Keywords}

Clonostachys rosea, Oxford Nanopore Technology, fungal genome, long-read sequencing

\section{Genome Announcement}

Plant pathogens cause destructive crop diseases leading to significant yield and quality decline. To counteract this, chemical pesticides are extensively used throughout the world, which has led to concerns about environmental pollution and human health. Biological control is an environmentally friendly alternative. Therefore, the use of biological control agents against plant pathogens can be expected to increase in future agricultural and horticultural production.

The necrotrophic mycotrophic fungus Clonostachys rosea, a member of the order Hypocreales in the class Sordariomycetes, is a highly versatile biological control agent that is reported to control diseases caused by a wide range of plant pathogenic fungi (Sun et al. 2020), such as Botrytis cinerea (Wang et al. 2019), Fusarium graminearum (Xue et al. 2008), and Sclerotinia sclerotiorum (Rodríguez et al. 2011). The biocontrol mechanisms of $C$. rosea against plant pathogenic fungi are primarily attributed to direct parasitism, secretion of fungal cell wall degrading enzymes, production of secondary metabolites such as antibiotics and toxins, and induction of plant resistance (Sun et al. 2020). Recently, the genome assembly of three C. rosea strains has been published (Broberg et al. 2018; Karlsson et al. 2015; Liu et al. 2016; Sun et al. 2015). The available genome resources are valuable for identifying biocontrol-related genes to improve the biological control ability of $C$. rosea. Nevertheless, these genome assemblies are fragmented and contain hundreds of contigs, thus losing value as reference genomes.

The C. rosea strain CanS41 reported here was initially isolated from healthy oilseed rape (Brassica napus) as an endophytic fungus in Hongshan District, Wuhan, China. Here, we de novo sequenced and assembled the C. rosea CanS41 genome using long-read sequencing by

\section{${ }^{\dagger}$ Corresponding author: H. Q. Liu; liuhuiquan@nwsuaf.edu.cn}

The author(s) declare no conflict of interest.

Accepted for publication 29 January 2021.

() 2021 The American Phytopathological Society 
Table 1. Summary of genome assembly statistics of different $C$. rosea strains

\begin{tabular}{|c|c|c|c|c|}
\hline & CanS41 & $67-1^{a}$ & YKD0085 $^{\mathrm{b}}$ & IK726 ${ }^{\mathrm{C}}$ \\
\hline Sequencing platform & Oxford Nanopore & Illumina HiSeq & Illumina MiSeq & Illumina + PacBio \\
\hline Assembly size (Mb) & 60.68 & 55.42 & 55.18 & 70.74 \\
\hline Read coverage depth & 230 & 150 & 31 & NA \\
\hline$\%$ GC content & 48.55 & 49.88 & 49.27 & 48.74 \\
\hline \# of contigs & 26 & 1,552 & 1,082 & 2,321 \\
\hline N50 contig (Mb) & 3.02 & 0.08 & 0.38 & 0.07 \\
\hline \# of gene models & 20,818 & 20,747 & 16,120 & 21,246 \\
\hline$\%$ repetitive DNA & 8.38 & 2.43 & 2.85 & $N A^{d}$ \\
\hline$\%$ BUSCO completeness & 100 & 99.6 & 100 & NA \\
\hline$\%$ k-mer completeness & 97.9 & NA & NA & NA \\
\hline Consensus QV score ${ }^{\mathrm{e}}$ & 42.45 & NA & NA & NA \\
\hline
\end{tabular}

${ }^{a}$ Reported by Sun et al. 2015.

${ }^{b}$ Reported by Liu et al. 2016.

${ }^{c}$ Reported by Broberg et al. 2018.

d NA, values unavailable due to the raw sequencing data or genome sequences (for IK726) not available at public databases.

${ }^{\mathrm{e}}$ Consensus quality $(\mathrm{QV})=-10 \log 10 E$ for a probability of error $E$ at each base in the assembly (Rhie et al. 2020). Q40 corresponds to $99.99 \%$ accuracy.

Oxford Nanopore Technologies (ONT) aiming to present a high-quality reference genome. Genomic DNA of CanS41 was extracted from pure cultured hyphae by the CTAB method. In total, 971,501 long-reads (14.13 Gb, 230x coverage) were generated from ONT platform. The mean length of reads is $14,547 \mathrm{bp}$ and the read length $\mathrm{N}_{50}$ is $17,955 \mathrm{bp}$. We performed read corrections, trimming, and genome assembly of CanS41 using Canu v. 1.7 (Koren et al. 2017). Redundant contigs in the primary assembly were removed using purge_dups v. 1.0.1 (Guan et al. 2020). The assembled genome was further polished by ONT reads using Racon v. 1.4.13 (Vaser et al. 2017) through three iterations. The final genome assembly of $C$. rosea strain CanS41 is $60.68 \mathrm{Mb}$ containing 26 contigs with an $\mathrm{N}_{50}$ of $3.02 \mathrm{Mb}$ (Table 1, Fig. 1). We estimated the baselevel accuracy and completeness of the genome assembly using Merqury software (Rhie et al. 2020) based on efficient k-mer set operations. The results revealed a consensus quality (QV) score of 42.45 (Q40 corresponds to $99.99 \%$ accuracy) and a k-mer completeness score of $97.9 \%$ (Table 1), suggesting that our genome assembly is highly accurate and complete.

De novo identification and modeling of repeat families in CanS41 were performed with RepeatModeler v. 1.0.11 (http://www.repeatmasker.org). Repeat elements were then annotated and classified by RepeatMasker v. 4.0 .9 (Tarailo-Graovac and Chen 2009). As a result, $8.38 \%$ of the CanS41 genome are repetitive DNA (Table 1) and $3.48 \%$ of the repeats are long terminal repeat retrotransposons (LTR-RT).

To call gene models from the genome sequences of $C$. rosea strain CanS41, we downloaded RNA-seq data of $C$. rosea during growth, development, and parasitic interaction with different plant pathogenic fungi under accession number PRJEB4200, PRJNA272346, PRJNA420967, and PRJNA540080. The RNA-seq reads were mapped to the CanS41 genome by HISAT2 v. 2.1.0 (Kim et al. 2015). We used BRAKER2 pipeline (Hoff et al. 2019) to perform gene model prediction with parameters '-fungus' and '-etpmode' by integrating the Augustus v. 3.3.3 (Stanke et al. 2008) and GeneMark-ES suite v. 4.48_3.60 (Ter-Hovhannisyan et al. 2008) software. RNASeq alignments and the fungi OrthoDB v. 9 database (Zdobnov et al. 2017) were supplied as transcript and protein evidence. In total, we identified 20,818 protein-coding genes (>50 aa) for CanS41. Assessing the gene annotation completeness using BUSCO (Simão et al. 2015) with the 'fungi_odb9' library followed by manual correction showed that all of the BUSCO genes were present in our predicted protein-coding gene set (Table 1). We then performed gene ontology (GO) functional annotation using InterproScan v. 5.45-80.0 (Jones et al. 2014) and assigned GO terms to 10,631 genes.

Carbohydrate-active enzymes (CAZymes) and secondary metabolites (SMs) fulfill the important functions for parasitizing, acquiring nutrition, and communicating between different species (Macheleidt et al. 2016; Walton 1994; Zhao et al. 2014). In total, 694 genes encoding CAZymes were identified by dbCAN (Yin et al. 2012). Among them, glycoside hydrolases (GHs) contribute more than half (387). We identified 66 SM gene clusters using AntiSMASH (Medema et al. 2011), including 36 nonribosomal peptide synthetases (NRPS), 32 polyketide synthases (PKS), 


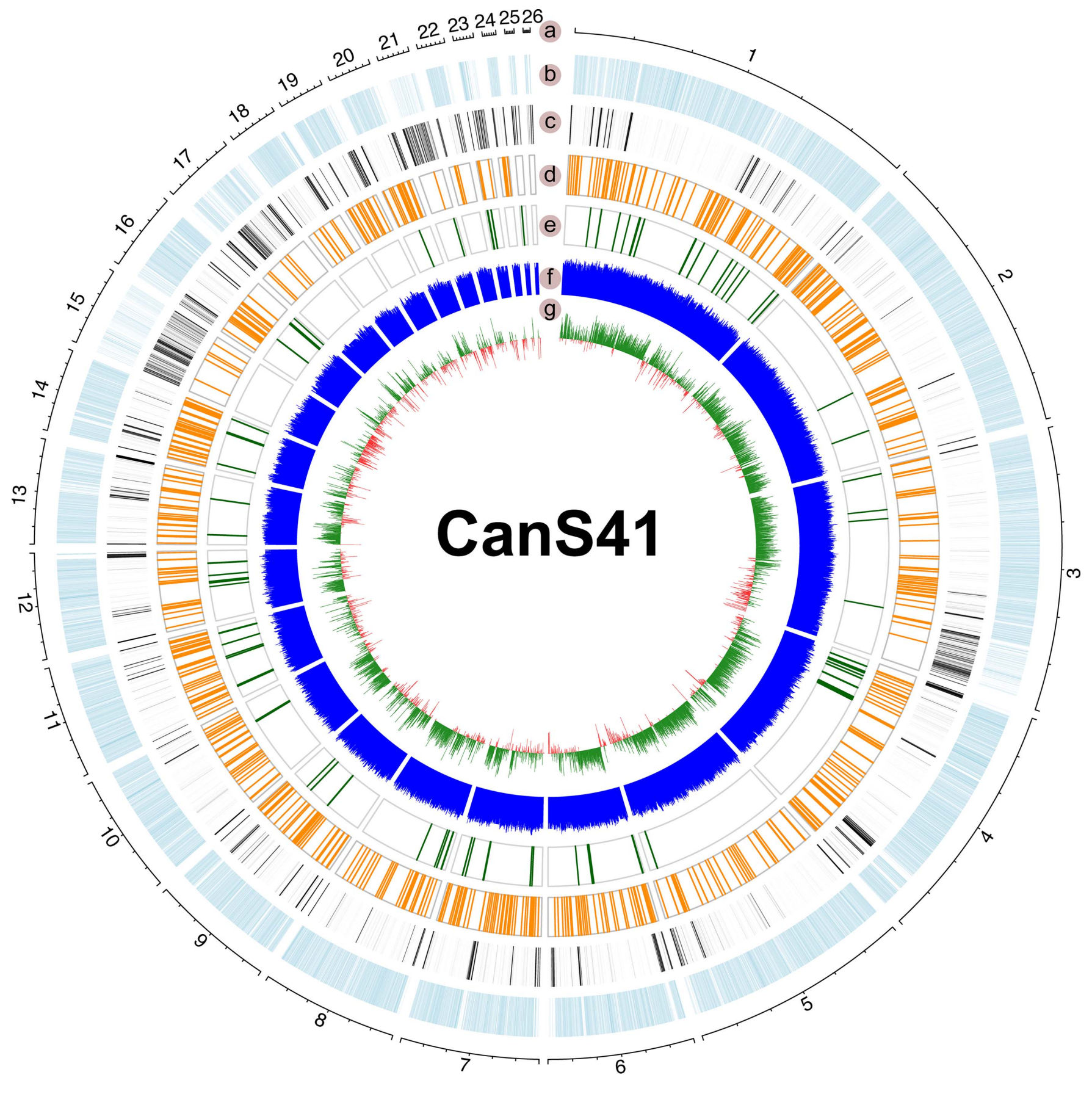

Fig. 1. Circos-plot of genomic features of $C$. rosea strain CanS41. a, Ideograms of all contigs of $C$. rosea CanS41; b, gene density heatmap; c, repetitive DNA density heatmap; $\mathbf{d}$, distribution of CAZyme genes; e, distribution of secondary metabolic (SM) backbone genes; $\mathbf{f}$, nanopore reads coverage histogram; $\mathbf{g}, \mathrm{GC}$ content plotted as the deviation from the average GC content of the entire genome.

15 NRPS-like, 10 terpenes, two indoles, and two betalactones. In addition, 2,099 proteins were predicted to be secretory proteins by SignalP-5.0 (Almagro Armenteros et al. 2019) and DeepLoc v. 1.0 (Almagro Armenteros et al. 2017). Among them, 469 genes were predicted to be putative effectors by EffectorP v. 2.0 (Sperschneider et al. 2018).

The genome assembly and gene annotation of $C$. rosea strain CanS41 were deposited at GenBank under the accession JADCTT000000000. The version described in this paper is JADCTT010000000. Nanopore long reads are available in the NCBI Sequenced Read Archive database under the accession number SRR12847398. 


\section{Literature Cited}

Almagro Armenteros, J. J., Sønderby, C. K., Sønderby, S. K., Nielsen, H., and Winther, O. 2017. DeepLoc: prediction of protein subcellular localization using deep learning. Bioinformatics 33:3387-3395.

Almagro Armenteros, J. J., Tsirigos, K. D., Sønderby, C. K., Petersen, T. N., Winther, O., Brunak, S., von Heijne, G., and Nielsen, H. 2019. SignalP 5.0 improves signal peptide predictions using deep neural networks. Nat. Biotechnol. 37:420-423.

Broberg, M., Dubey, M., Sun, M.-H., Ihrmark, K., Schroers, H.-J., Li, S.-D., Jensen, D. F., Brandström Durling, M., and Karlsson, M. 2018. Out in the cold: identification of genomic regions associated with cold tolerance in the biocontrol fungus Clonostachys rosea through genome-wide association mapping. Front. Microbiol. 9:2844.

Guan, D., McCarthy, S. A., Wood, J., Howe, K., Wang, Y., and Durbin, R. 2020. Identifying and removing haplotypic duplication in primary genome assemblies. Bioinformatics 36:2896-2898.

Hoff, K. J., Lomsadze, A., Borodovsky, M., and Stanke, M. 2019. Whole-genome annotation with BRAKER. Methods Mol. Biol. 1962:65-95.

Jones, P., Binns, D., Chang, H.-Y., Fraser, M., Li, W., McAnulla, C., McWilliam, H., Maslen, J., Mitchell, A., Nuka, G., Pesseat, S., Quinn, A. F., Sangrador-Vegas, A., Scheremetjew, M., Yong, S.-Y., Lopez, R., and Hunter, S. 2014. InterProScan 5: genome-scale protein function classification. Bioinformatics 30:12361240.

Karlsson, M., Durling, M. B., Choi, J., Kosawang, C., Lackner, G., Tzelepis, G. D., Nygren, K., Dubey, M. K., Kamou, N., Levasseur, A., Zapparata, A., Wang, J., Amby, D. B., Jensen, B., Sarrocco, S., Panteris, E., Lagopodi, A. L., Pöggeler, S., Vannacci, G., Collinge, D. B., Hoffmeister, D., Henrissat, B., Lee, Y.-H., and Jensen, D. F. 2015. Insights on the evolution of mycoparasitism from the genome of Clonostachys rosea. Genome Biol. Evol. 7:465-480.

Kim, D., Langmead, B., and Salzberg, S. L. 2015. HISAT: a fast spliced aligner with low memory requirements. Nat. Methods 12:357-360.

Koren, S., Walenz, B. P., Berlin, K., Miller, J. R., Bergman, N. H., and Phillippy, A. M. 2017. Canu: scalable and accurate long-read assembly via adaptive $k$-mer weighting and repeat separation. Genome Res. 27:722-736.

Liu, S., Chang, Y., Hu, X., Gong, X., Di, Y., Dong, J., and Hao, X. 2016. Draft genome sequence of fungus Clonostachys rosea strain YKD0085. Genome Announc. 4:e00538-16.

Macheleidt, J., Mattern, D. J., Fischer, J., Netzker, T., Weber, J., Schroeckh, V., Valiante, V., and Brakhage, A. A. 2016. Regulation and role of fungal secondary metabolites. Annu. Rev. Genet. 50:371-392.

Medema, M. H., Blin, K., Cimermancic, P., de Jager, V., Zakrzewski, P., Fischbach, M. A., Weber, T., Takano, E., and Breitling, R. 2011. antiSMASH: rapid identification, annotation and analysis of secondary metabolite biosynthesis gene clusters in bacterial and fungal genome sequences. Nucleic Acids Res. 39 (suppl_2):W339-W346.
Rhie, A., Walenz, B. P., Koren, S., and Phillippy, A. M. 2020. Merqury: referencefree quality, completeness, and phasing assessment for genome assemblies. Genome Biol. 21:245.

Rodríguez, M. A., Cabrera, G., Gozzo, F. C., Eberlin, M. N., and Godeas, A. 2011. Clonostachys rosea BAFC3874 as a Sclerotinia sclerotiorum antagonist: mechanisms involved and potential as a biocontrol agent. J. Appl. Microbiol. 110:11771186.

Simão, F. A., Waterhouse, R. M., loannidis, P., Kriventseva, E. V., and Zdobnov, E. M. 2015. BUSCO: assessing genome assembly and annotation completeness with single-copy orthologs. Bioinformatics 31:3210-3212.

Sperschneider, J., Dodds, P. N., Gardiner, D. M., Singh, K. B., and Taylor, J. M. 2018. Improved prediction of fungal effector proteins from secretomes with EffectorP 2.0. Mol. Plant Pathol. 19:2094-2110.

Stanke, M., Diekhans, M., Baertsch, R., and Haussler, D. 2008. Using native and syntenically mapped cDNA alignments to improve de novo gene finding. Bioinformatics 24:637-644.

Sun, Z.-B., Li, S.-D., Ren, Q., Xu, J.-L., Lu, X., and Sun, M.-H. 2020. Biology and applications of Clonostachys rosea. J. Appl. Microbiol. 129:486-495.

Sun, Z.-B., Sun, M.-H., and Li, S.-D. 2015. Draft genome sequence of mycoparasite Clonostachys rosea strain 67-1. Genome Announc. 3:e00546-15.

Tarailo-Graovac, M., and Chen, N. 2009. Using RepeatMasker to identify repetitive elements in genomic sequences. Curr. Protoc. Bioinforma. 25:4.10.1-4.10.14.

Ter-Hovhannisyan, V., Lomsadze, A., Chernoff, Y. O., and Borodovsky, M. 2008. Gene prediction in novel fungal genomes using an ab initio algorithm with unsupervised training. Genome Res. 18:1979-1990.

Vaser, R., Sović, I., Nagarajan, N., and Šikić, M. 2017. Fast and accurate de novo genome assembly from long uncorrected reads. Genome Res. 27:737-746.

Walton, J. D. 1994. Deconstructing the cell wall. Plant Physiol. 104:1113-1118.

Wang, Q., Chen, X., Chai, X., Xue, D., Zheng, W., Shi, Y., and Wang, A. 2019. The involvement of jasmonic acid, ethylene, and salicylic acid in the signaling pathway of Clonostachys rosea-induced resistance to gray mold disease in tomato. Phytopathology 109:1102-1114.

Xue, A., Chen, Y., Voldeng, H., Savard, M., and Tian, X. 2008. Biological control of Fusarium head blight of wheat with Clonostachys rosea strain ACM941. Cereal Res. Commun. 36(Suppl. 6):695-699.

Yin, Y., Mao, X., Yang, J., Chen, X., Mao, F., and Xu, Y. 2012. dbCAN: a web resource for automated carbohydrate-active enzyme annotation. Nucleic Acids Res. 40 (W1):W445-W451.

Zdobnov, E. M., Tegenfeldt, F., Kuznetsov, D., Waterhouse, R. M., Simão, F. A., Ioannidis, P., Seppey, M., Loetscher, A., and Kriventseva, E. V. 2017. OrthoDB v9.1: cataloging evolutionary and functional annotations for animal, fungal, plant, archaeal, bacterial and viral orthologs. Nucleic Acids Res. 45(D1):D744-D749.

Zhao, Z., Liu, H., Wang, C., and Xu, J.-R. 2014. Correction: Comparative analysis of fungal genomes reveals different plant cell wall degrading capacity in fungi. BMC Genomics 15:6. 